\title{
Ultrasound Elastography in Liver Tissue: Current Status
}

\author{
Mingzhu Zhang, MD ${ }^{a}$, Zhaoyan Ding, MD ${ }^{a}$, Xiaoyan Niu, MD ${ }^{a}$, Yuxiu Gao, MD ${ }^{a}$, Cheng Zhao, MD ${ }^{a, *}$ \\ ${ }^{a}$ Department of Ultrasound, The Affiliated Hospital of Qingdao University, Qingdao, China. \\ Received May 24, 2021; revision received June 20, 2021; accepted July 02, 2021
}

\begin{abstract}
Chronic liver disease is common in China and worldwide, with liver fibrosis as the primary pathological finding. Any chronic liver disease can lead to hepatic fibrosis and gradually develop into cirrhosis. Complications, such as portal hypertension, gastrointestinal bleeding, and liver failure can occur. Some patients even develop hepatocellular carcinoma. Thus, timely diagnosis of hepatic fibrosis is vital for the assessment of etiology, treatment, and prognosis. Conventional ultrasound imaging shows low sensitivity with its subjectivity for preliminary diagnosis of liver fibrosis, creating limitations in qualitative and quantitative evaluation. Ultrasound elastography is a recently developed technique that can help overcome these limitations. The elastic imaging method combines conventional ultrasound to assess liver stiffness along with routine examination. This "one-stop" check for liver disease opens new prospects for clinical and scientific research and improves the accuracy of disease diagnosis for broad clinical application. This article will review the current status of ultrasound elastography for its applications in chronic liver diseases.
\end{abstract}

Key words: Chronic liver disease; Ultrasound; Shear wave elastography; Transient elastography; Point shear wave elastography; Two-dimensional shear wave elastography

Advanced Ultrasound in Diagnosis and Therapy 2021; 03: 197-203

DOI: 10.37015/AUDT.2021.210014

\section{Overview of Ultrasound Elastography}

Ultrasound elastography was first proposed by Ophir et al in 1991 [1]. The technique has been used for nearly 30 years and is now widely applied in clinical practice since it was proposed. Elasticity refers to the ability of an object to recover its original shape and size after alteration by an external force. Strain refers to the degree to which the size and shape of an object changes in response to an external force. Ultrasound elastography differs from traditional ultrasound imaging although it is based on the conventional two-dimensional ultrasound imaging. The operator manually exerts pressure on the tissue through the probe to cause deformation and displacement, recorded as a color spectrum (elastogram). By comparing color differences of images when pressure is applied, the differences between stiffness of lesions and the surrounding tissues are evaluated. Thus, corresponding elasticity values can be obtained, and provide helpful information for diagnosing of lesions [2]. At present, this technique is widely used to assess organ structures, such as thyroid, breast, liver, kidney, and prostate.

Technical innovation in elastography continuously evolved over the years. Sarvazyan et al. proposed shear wave elastography in 1998 [3]. It was an imaging method that classified its methods according to measurement results. The elastic imaging can be created by applying an internal or external dynamic, quasi-static, or static excitation to tissues and organs to cause deformation. Subsequently, signal processing produces a quantitative image that reflects the elasticity coefficient as a measure of stiffness. Thus, the coefficient directly or indirectly reflects mechanical differences within tissues, and elastograms illustrate local strain, i.e., Young's moduli or Poisson's ratios. Elasticity parameters are not directly related to ultrasound parameters, but Young's moduli

\footnotetext{
* Corresponding author: Department of Ultrasound, The Affiliated Hospital of Qingdao University, China.

e-mail: zhaochengdr@163.com unrestricted use, distribution and reproduction in any medium provided that the original work is properly attributed.
} 
can be quantitatively measured, so called shear wave elastography (SWE) $[4,5]$.

Ultrasound-based shear wave elastography is a new non-invasive imaging technology. It is real-time, rapid, noninvasive, easy to control and gives qualitative and quantitative information on tissue stiffness. These advantages eliminate deficiencies of traditional ultrasound imaging, expand the technical field of ultrasound, improve the accuracy of disease diagnosis. These suggest ultrasound-based shear wave elastography has a broad application prospect and clinical value [6]. Shear wave velocity varies among tissues and organs. Stiffness is measured by sending low-frequency waves through the body. Further, the velocity of propagation is directly related to Young's moduli; the faster the propagation, the higher the modulus. Tissues are of the different stiffness values, thus can be measured elastic differences between different organizations [7]. Shear wave elastography mainly includes transient elastography(TE), point shear wave elastography (pSWE) and two-dimensional shear wave elastography (2D-SWE).

\section{Application in Liver Tissue}

With fibrosis as the primary pathological finding, chronic liver disease is a significant public health problem worldwide [8]. Liver fibrosis is caused by recurrent or persistent liver inflammation. The process is a dynamic and asymptotic accumulation of fibrous tissue. Essentially, any chronic liver disease can lead to liver fibrosis, gradually progress to cirrhosis, and even develop into hepatocellular carcinoma (HCC). The degree of fibrosis is an independent risk factor for HCC, and is not irreversible [7]. Therefore, early detection of fibrosis and determining the degree of accumulation is crucial for treatment and prognosis.

The main clinical methods for diagnosis of liver diseases include imaging, blood biochemistry, and tissue biopsy. The biopsy is the current "gold standard" for diagnosis of liver disease. The technique is invasive and can cause bleeding, pain, perforation, or other complications, which are not generally acceptable. Further, the amount of tissue collected is limited, accounting for about $1 / 50,000$ of total liver volume. Thus, sampling error rate can reach $10 \%-45 \%$ [9]. Further, both the size and location of the sample and subjective differences among pathologists impact the assessment of biopsies. In addition, radiation is used in many imaging methods, which cannot be used in some patients, such as children and pregnant women. These shortcomings facilitate the development of methods for noninvasive liver examination.
Conventional two-dimensional ultrasound is easy to administer, but shows limited sensitivity for preliminary diagnosis of liver fibrosis. For example, irregular liver margins, thickening and strengthening of internal echoes are challenging to discern. Further, low sensitivity and strong subjectivity limit accuracy in qualitative and quantitative diagnoses. Finally, differential diagnosis of benign and malignant nodules and changes in tissue stiffness cannot be evaluated. Thus, quantifying the degree of fibrosis and improving the accuracy of diagnosis by shear wave elastography is critical. This method provides a new approach for diagnosing liver diseases, differentiating benign and malignant nodules, and improving the detection rate of spaceoccupying hepatic lesions. The use of the method should prove helpful for the early diagnosis and treatment of liver fibrosis. Also, puncture biopsy success rate and satisfaction with such sampling under the guidance of elastography are better than that under the guidance by conventional ultrasound in liver tissue [10].

Contrast-enhanced ultrasound and shear wave elastography can be used to assess the degree of fibrosis. $\mathrm{Wu}$ et al. indicated that both angiography and shear wave elastography show high diagnostic accuracy with higher accuracy of shear wave elastography [11]. The latter technique has gradually become an essential method for quantifying fibrosis and thus guide the assessment of prognosis and follow-up monitoring of patients with chronic liver disease. Shear wave propagation velocity is different between diffuse liver lesions and focal lesions. Thus, elastography can be used to distinguish benign from malignant tumors and discriminate among tumor types. These capabilities improve the accuracy of clinical diagnosis.

\section{Transient Elastography (TE)}

\section{Principle}

TE belongs to the shear wave velocity measurement technology, which uses surface mechanical forces for imaging rather than acoustic radiation forces. The propagation velocity of shear waves can be measured without generating images [5]. Low-frequency mechanical vibration from a special probe causes displacement and deformation of tissue structure that produces shear waves. The method measures propagation velocity of the shear wave and displays it as an elastic modulus, then calculates tissue stiffness based on velocity $(\mathrm{kPa})$. Measured velocity is directly related to stiffness; the faster the shear wave propagates, the greater the tissue stiffness. 


\section{Clinical application}

Colombo et al. described elastography performed on normal human liver using the fibroscan with the TE values ranged from 4.4 to $5.5 \mathrm{kPa}$ [12]. Sharma et al. suggested that TE could be used to distinguish patients with acute hepatitis from patients with chronic acute liver failure. Specificity and sensitivity could reach above 90\% [13]. Mobarak et al. indicated that TE had higher diagnostic value than other examination methods for diagnosis of liver fibrosis progression and cirrhosis [14]. TE measures the degree of liver fibrosis accurately and can play an important role in predicting complications of advanced compensated chronic liver disease (cACLD), such as gastroesophageal varices, portal hypertension, cirrhosis, and HCC [15]. Occurrence and progression of HCC are highly correlated with severe fibrosis and decompensation of the liver [8]. TE can also be used to evaluate the possibility of HCC. TE thus assists clinicians in selecting eligible patients for medication research and clinical trials.

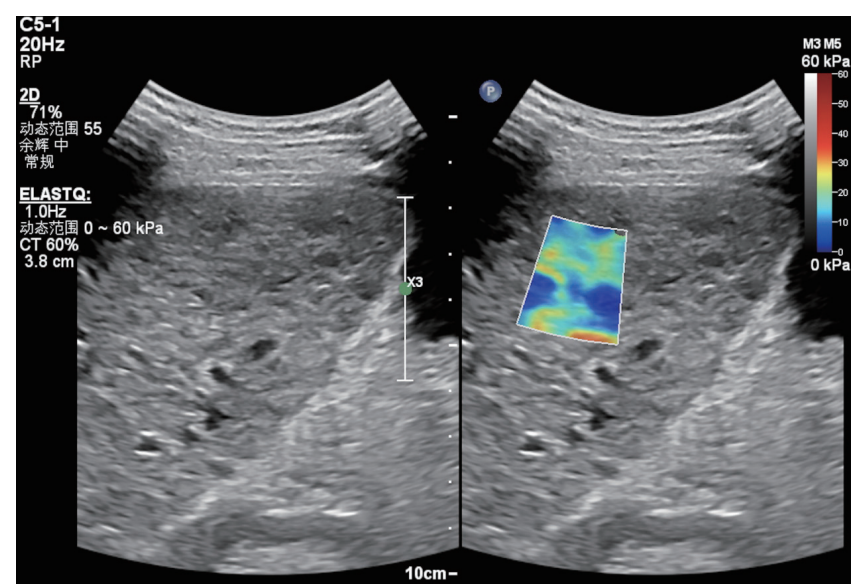

Figure 1 TE images in a 33-year-old man with a history of hepatitis B. Transient elastography of the liver performs with the Philips EPIQ 7 system through intercostal access. The measurement is given in $\mathrm{kPa}$. The hardness of the liver is $15.2 \mathrm{kPa}$, which is above the normal range.

\section{Advantages and disadvantages}

TE technology is mature and has been used in clinical examination for many years. Moreover, a standardized reporting system has been developed that is rapid, high accuracy, strong repeatability, and quantitative analysis. TE for liver diagnosis is an early elastography application, and has been reported in many studies. The relevant technology has become mature, and its value in the clinic is widely recognized and affirmed by clinicians and patients. Compared with other elastography methods, TE improves stiffness assessment accuracy and sensitivity by reducing the effect of shear waves induced by the low-frequency vibration on the diffraction phenomenon, thus improving imaging effect. Moreover,
TE acquisition is rapid, usually $<100 \mathrm{~ms}$, and is suitable for hepatic applications. Liver stiffness is directly related to shear wave propagation speed; when the propagation speed is faster, the hardness of liver tissue will be greater [16]. Finally, TE measures a volume of liver tissue 100 times more than the sample size of a typical biopsy [17]. Thus, TE is more reproducible and representative.

Conversely, TE also has certain limitations. Separate instruments and probes are needed for elastography. Moreover, it cannot be simultaneously checked with conventional ultrasound. These limitations increase examination cost and reduce convenience [5]. TE is a one-dimensional ultrasonic elastography technology that achieves only standardized measurements and thus cannot locate the position of lesions on two-dimensional images in real-time. Also, it is difficult to avoid intrahepatic bile ducts, blood vessels, gallbladder, and other non-target structures during the measurements.

TE sampling range is limited and about $20 \%$ of patients may show unreliable or failing test results [18]. Additionally, TE has limited ability to distinguish mild liver fibrosis, and limited sampling depth may produce significant measurement errors of stiffness in some situations, such as in patients with severe liver atrophy, abdominal ascites, narrow intercostal space, or high body mass index (BMI) [19].

\section{Point Shear Wave Elastography (pSWE)}

\section{Principle}

pSWE is also known as acoustic radiation force impulse (ARFI) quantitative detection technology, and measures shear wave velocity. pSWE uses an ordinary low-frequency ultrasound probe to transmit acoustic radiation force pulses to the region of interest (ROI). The pulse is focused at a certain depth of the tissue. The corresponding tissue is displaced, deformed, and shear waves are generated. Shear wave velocity $(\mathrm{SWV}, \mathrm{m} / \mathrm{s})$ at the ROI is recorded and may be converted into an elastic modulus value. This texture information can be used to quantify tissue stiffness.

\section{Clinical application}

pSWE is widely used for the diagnosis of parenchymal diseases in abdominal organs. Specially, this use has significant value for measuring tissue fibrosis in the liver, which is useful for diagnosis, treatment, and prognosis of chronic liver disease. Measured values of pSWE in normal liver range from 0.94 to $1.45 \mathrm{~m} / \mathrm{s}$ [20]. Furthermore, SWV is related to the stage of liver fibrosis, and higher SWV is associated with harder tissue [21]. The core of pSWE technology is virtual touch tissue imaging (VTTI) and virtual touch tissue and 
quantification (VTTQ). This newly developed virtual touch tissue imaging quantification (VTIQ) technology has also been used for qualitative and quantitative analysis of tissue stiffness in clinical practice.

pSWE as a noninvasive method is used to predict the degree of liver fibrosis. When elastography was performed on healthy people and patients with mild or moderate liver fibrosis confirmed by liver biopsy as chronic liver disease, stiffness of left liver tissue was higher than that of right liver tissue; and when elastography was observed in patients with advanced liver fibrosis and cirrhosis, there was no obvious difference between liver lobes [22]. The left liver lobe is close to the heart and gastrointestinal tract, and is susceptible to cardiovascular fluctuations and gastrointestinal peristalsis. It also lacks shielding by the ribs, and the right liver is shielded by ribs. All these factors could affect liver elasticity [23]. Thus, the right lobe of the liver is more representative of actual elasticity. Right lobe values are more reliable and correlate better with the stage of liver fibrosis.

Lupsor et al. reported that TE and pSWE are equally accurate in diagnosing chronic viral hepatitis [24]. Dillman et al. suggest that VTIQ is important for grading hepatic fibrosis, which shows diagnostic significance for the degree of fibrosis and inflammation, and diagnosis results by these two techniques are highly correlated ( $\mathrm{r}$ $=0.79, \mathrm{P}<0.01$ ) [25]. pSWE technology was used to systematically analyze liver space-occupying lesions and showed the capability to distinguish benign from malignant lesions, with a sensitivity and specificity of $85 \%$ and $84 \%$, respectively [26].

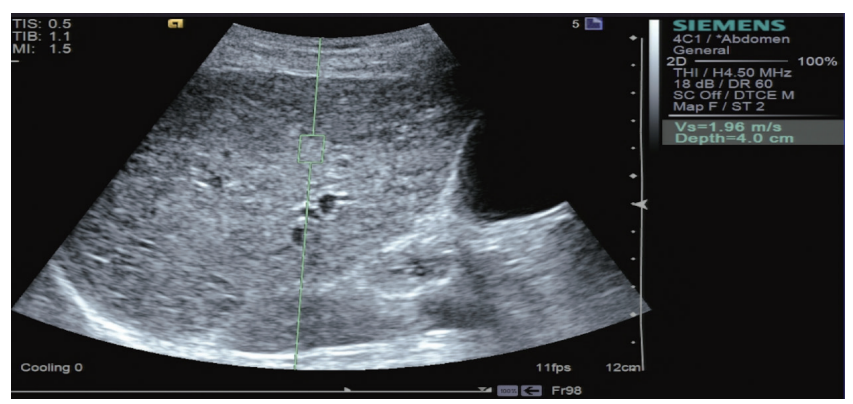

Figure 2 pSWE images in a 63-year-old man with a history of hepatitis B. pSWE of the liver performs with the Siemens system through intercostal access. The measurement is given in meters per seconds. The hardness of the liver is $1.96 \mathrm{~m} / \mathrm{s}$, which is above the normal range.

\section{Advantages and disadvantages}

pSWE do not need a special ultrasound probe, which is more easily operated and reproducible than TE, so it can be realized on the routine ultrasound diagnosis system. The reliability and success rate of pSWE for quantitative detection of liver fibrosis are higher than for
TE, but with no significant difference in the diagnostic efficacy. pSWE, also known as acoustic biopsy, uses real-time ultrasound elastography to solve problems in 2D images. Doppler ultrasound imaging, real-time two-dimensional elastography, and contrast-enhanced ultrasound can be combined to evaluate hepatic lesions and measure liver stiffness within a defined range. pSWE can also avoid the influence of non-target structures and produces better visualization output that allows multidirection and multi-angle measurement of different parts of the liver. Under 2D ultrasound guidance, pSWE can select ROI more accurately, reduce sampling error, and make detection results more accurate and intuitive [6]. These characteristics can provide the value of diagnostic information for the disease and provide more comprehensive evidences for clinicians in diagnosis and treatment.

The shear wave generated by pSWE is less affected by peritoneal effusion and can be used in patients with this condition [27]. Obesity shows some influence on results, but the degree of influence is less than that for TE. Thus, pSWE can be used in patients with high BMI. Local acoustic radiation energy is relatively large and sampling depth is limited to about $4-5 \mathrm{~cm}$. Sampling volume is limited $(10 \mathrm{~mm} \times 6 \mathrm{~mm})$, and the method is susceptible to fluctuations in abdominal great vessels and gas [28].

\section{Two-Dimensional Shear Wave Elastography}

\section{Principle}

2D-SWE is most commonly used shear wave elastography technology. The principle of 2D-SWE is similar to that of TE. However, it effectively overcomes deficiencies in TE imaging. Common ultrasound diagnosis system can realize 2D-SWE without special ultrasound equipment. The probe emits acoustic radiation pulses to the examined tissues at high speed. Pulses continuously focus and excite tissues at different depths along the direction of the acoustic axis, causing tissues to vibrate and shift almost simultaneously. SWE uses the "Mach cone" phenomenon and Sonic Touch patented technology to generate a unique shear wave source at the focus location, resulting in significantly higher amplitude and longer propagation distance of the shear wave. The efficiency of shear wave generation is $4 \sim 8$ times that of a typical shear wave source [29]. The shear wave is detected by ultra-high-speed imaging technique with powerful computer. Simultaneously, SWV is quantitatively and accurately recorded in realtime, and a two-dimensional image of shear wave velocity is displayed using color-coding technology. Young's moduli $(\mathrm{kPa})$ can also be calculated with 
software included with the ultrasound equipment. SWV is positively correlated with Young's moduli. Both SWE and SWV measurements reflect the absolute stiffness of the corresponding tissues. The faster SWV is, the higher Young's moduli will be, and the stiffness of tissues will be. This technique has been widely and successfully used for inspection, diagnosis, and prognosis assessment of diseases in various organs, such as thyroid, breast, liver, prostate, musculoskeletal, and other tissues.

\section{Clinical application}

SWE can be used for clinical observation of liver. The main clinical sign of chronic liver disease is fibrosis [30]. Before elastographic examination, patients should fast for 4-6 h. During the examination, patients should be supine with the right upper limb lifted to widen the intercostal space, and the probe should be placed in the right intercostal space. Samir et al. demonstrated that the degree of liver fibrosis had the best correlation with stiffness of S5 and S6 segments of liver right lobe [31]. The sampling frame is placed $4-5 \mathrm{~cm}$ below the liver capsule to avoid larger liver blood vessels, the hepatic biliary duct, gallbladder, ribs, artifacts, and other nontarget structures. Patients hold their breath for $3-5 \mathrm{~s}$ in a calm breathing state. Deep inhalation, deep exhalation, or Valsalva maneuvers can increase pressure on liver tissue, which increase wave amplitude and produce inaccurate results. After elastography with the sampling frame is stabilized, the image is frozen, and the collected quantitative measurements are the best. The ROI, with a diameter of up to $2 \mathrm{~cm}$, is used to measure Young's moduli $(\mathrm{kPa})$. Measurements are repeated 10 times at the same location and the median value calculated. The standard for qualified data is color filling of elastic sampling frame more than $1 / 2$. Also, the minimum value of detection result is not 0 , and the ratio of interquartile space to median is less than $30 \%$.

Young's modulus is susceptible to the influence of bilirubin and transaminase and is positively correlated with its level [32]. This is means that Young's modulus is affected to a certain extent by related liver function. It should be point out that results also vary when different facilities or probes are used to measure liver stiffness. 2D-SWE is highly effective in diagnosing liver fibrosis, in which results are not affected by the size of the ROI, age, or BMI [33]. However, the depth of the sampling frame and different segments of the liver show significant influences. A typical 2D-SWE value for normal liver tissue is $5.1 \pm 1.0 \mathrm{kPa}$. The success rate for detection of liver fibrosis is higher for 2D-SWE than that of TE, and diagnostic efficiency is higher than either that of TE or pSWE. Detection success rate of 2D-SWE can reach $98.2 \%-98.9 \%$ [34].

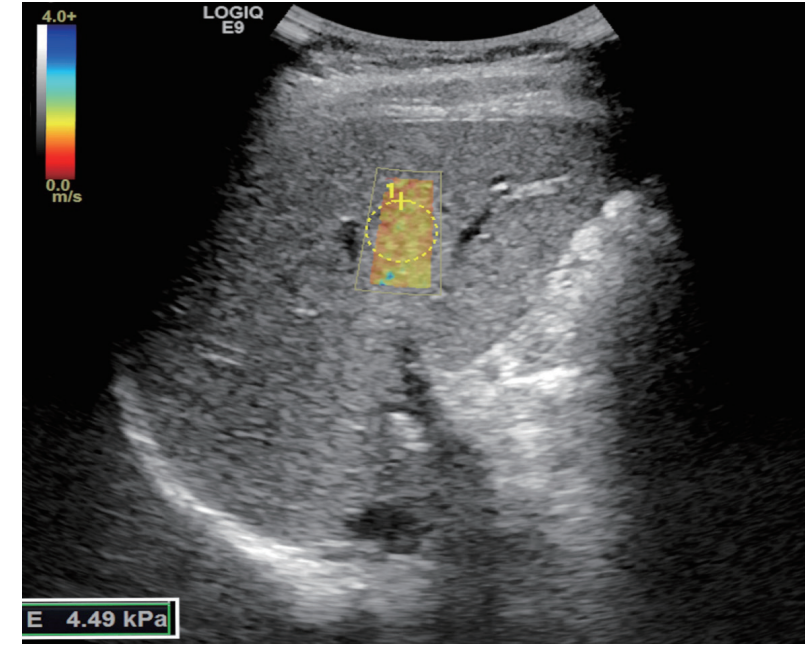

Figure 3 2D-SWE image in a 25-year-old woman in good health 2D-SWE of the liver performed with the GE LOGIQ E9 system through intercostal access. The measurement is given in $\mathrm{kPa}$. Image demonstrated one of the 10 measurements obtained in the same location. The hardness of the liver is $4.49 \mathrm{kPa}$, which is within the normal range.

\section{Advantages and disadvantages}

SWE combines conventional two-dimensional realtime ultrasonic images with stiffness information of the tissues. With two-dimensional ultrasonic imaging, nontarget structures such as hepatobiliary duct and gallbladder can be avoided making detection simpler and easier to interpret.

Furthermore, the anatomical position of tissue can be perfectly combined with stiffness to obtain color elastograms that visually display variations in liver stiffness. This rich information improves the accuracy of diagnosis. SWE is also highly sensitive for assessing the degree of liver fibrosis [35]. For patients with focal liver lesions, SWE can distinguish between pseudotumors, such as focal fatty infiltration, and true tumors, and detect lesions that are not easily found with two-dimensional ultrasound [36]. SWE has advantages of simple operation, high repeatability, rapid imaging, and a high quantitative measurement success rate. Moreover, 2D-SWE allows a large sampling area that can be rapidly changed to reduce sampling error on the basis of 2D-ultrasound. The technique is subject to few interference factors, which is highly representative and displays a wide application range. 2D-SWE can be used in patients with abdominal effusion, narrow intercostal space, and obesity. However, the success rate is low in patients that require dyspnea control. 2D-SWE can avoid high biological efficiency by focusing the acoustic radiation force in a single place, making imaging safer and more reliable. However, the stage of liver fibrosis is not well defined, which few prospective studies are available and relevant literature is lacking [37]. 
The accuracy of shear wave measurement can be affected in patients with heart failure, excessive alcohol intake, cholestasis, increased central venous pressure, inflammation, and congestion. These conditions may lead to high measurement results [38].

\section{Conclusion}

Measurement of tissue stiffness using TE, pSWE, and 2D-SWE techniques should be performed by an experienced sonographer. Imaging principles and thresholds are different, as are units of expression, but all can assess stiffness of liver tissues by measuring the velocity of the liver shear wave propagation, which reflects the degree of liver fibrosis. Fibrosis increases the propagation speed of shear waves and Young's moduli. The stiffess of liver is statistically different by comparing the different periods of liver fibrosis.

As a new imaging technology, ultrasound shear wave elastography has many advantages and provides a practical new method for diagnosis, treatment, and prognosis of liver diseases. It is widely used in clinical practice. Stiffness of diseased tissues can be studied qualitatively and quantitatively, which overcomes the limitations of traditional ultrasonic imaging, improves the accuracy of diagnosis, and optimizes the tumor classification. It is used as an extension of conventional ultrasonic examination and is expected to become routine for evaluation of liver fibrosis. However, ultrasound shear wave elastography is still affected by several factors in practical clinical applications, such as operator proficiency and the presence of inflammation and cholestasis.

In conclusion, more in-depth clinical research on shear wave imaging should expand to incorporate multietiological and multi-center large-scale studies. Such research can produce a comprehensive and efficient new diagnostic model for ultrasound shear wave elastography that can identify pathological changes in liver tissue more objectively and accurately. Such a method can provide more powerful help for the selection of clinical treatment options.

\section{Conflicts of Interest}

The authors declare no conflicts of interest.

\section{References:}

[1] Ophir J, Alam SK, Garra BS, Kallel F, Konofagou EE, Krouskop T, et al. Elastography: Imaging the elastic properties of soft tissues with ultrasound. J Med Ultrason 2002; 29:155.

[2] Ricci P, Maggini E, Mancuso E, Lodise P, Cantisani V, Catalano C. Clinical application of breast elastography: state of the art. Eur $J$ Radiol 2014; 83: 429-437.

[3] Sarvazyan AP, Rudenko OV, Swanson SD, Fowlkes JB, Emelianov
SY. Shear wave elasticity imaging: a new ultrasonic technology of medical diagnostics. Ultrasound Med Biol 1998; 24: 1419-1435.

[4] Bamber J, Cosgrove D, Dietrich CF, Fromageau J, Bojunga J, Calliada F, et al. EFSUMB guidelines and recommendations on the clinical use of ultrasound elastography. Part 1: Basic principles and technology. Ultraschall Med 2013; 34: 169-184.

[5] Zhi X, Qian XL, Geng HY, Hu XD. Advances in clinical application of shear wave elastography. China Medical Equipment 2016; 12: 6670 .

[6] Zheng RQ, Jin JY. Advances in the application of ultrasonic shear wave elastography in liver diseases. Organ Transplantation 2017; 4 260-266.

[7] Chon YE, Jung ES, Park JY, Kim DY, Ahn SH, Han KH, et al. The accuracy of noninvasive methods in predicting the development of hepatocellular carcinoma and hepatic decompensation in patients with chronic hepatitis B. Journal of Clinical Gastroenterology 2012; 46: 518-525.

[8] Muller M, Gennisson JL, Deffieux T, Tanter M, Fink M. Quantitative viscoelasticity mapping of human liver using supersonic shear imaging: preliminary in vivo feasibility study. Ultrasound Med Biol 2009; 35: 219-229.

[9] Seeff LB, Everson GT, Morgan TR, Curto TM, Lee WM, Ghany $\mathrm{MG}$, et al. Complication rate of percutaneous liver biopsies among persons with advanced chronic liver disease in the HALT-C trial. Clin Gastroenterol Hepatol 2010; 8: 877-883.

[10] Lu YP, Han W, Xiao XQ. Application value of two-dimensiona ultrasound and ultrasound elastography in differentiating benign and malignant liver lesions. Chinese Journal of Liver Diseases 2017; 9: 45-49.

[11] Wu TN, Fu SQ, Liu BY, Wang DL, Tan LL. Efficacy of ultrasound elastography and contrast imaging in the evaluation of liver fibrosis in chronic hepatitis B. The Journal of Practical Medicine 2017; 33: 3983-3986.

[12] Colombo S, Belloli L, Zaccanelli M, Badia E, Jamoletti C, Buonocore M, et al. Normal liver stiffness and its determinants in healthy blood donors. Dig Liver Dis 2011; 43: 231-236.

[13] Sharma P, Bansal R, Matin A, Tyagi P, Bansal N, Singla V, et al. Role of transient elastography (Fibroscan) in differentiating severe acute hepatitis and acute on chronic liver failure. J Clin Exp Hepatol 2015; 5: 303-309.

[14] Mobarak L, Nabeel MM, Hassan E, Omran D, Zakaria Z. Real-time elastography as a noninvasive assessment of liver fibrosis in chronic hepatitis C Egyptian patients: a prospective study. Ann Gastroenterol. 2016; 29: 358-362.

[15] Zhang X, Wong GL, Wong VW. Application of transient elastography in nonalcoholic fatty liver disease. Clin Mol Hepatol 2020; 26: 128141.

[16] Ge N, He Q. Evaluation of grade of liver fibrosis by ultrasound elastography. Journal of Clinical Hepatology 2016; 12: 2379-2382.

[17] Castera L, Forns X, Alberti A. Non-invasive evaluation of liver fibrosis using transient elastography. J Hepatol 2008; 48: 835-847.

[18] Zheng J, Zeng J, Zheng RQ, Wu T, Jin JY, Guo HY, et al. Comparison of efficacy of two-dimensional shear wave elastography, instantaneous elastography and real-time tissue elastography in the diagnosis of liver fibrosis. Chinese Journal of Medical Ultrasound 2016; 13: 496-500.

[19] Poynard T, Munteanu M, Luckina E, Perazzo H, Ngo Y, Royer L, et al. Liver fibrosis evaluation using real-time shear wave elastography: applicability and diagnostic performance using methods without a gold standard. J Hepatol 2013; 58: 928-935.

[20] Madhok R, Tapasvi C, Prasad U, Gupta AK, Aggarwal A. Acoustic radiation force impulse imaging of the liver: measurement of the 
normal mean values of the shearing wave velocity in a healthy liver. J Clin Diagn Res 2013; 7: 39-42.

[21] Yang L, Yuan JJ, Wang Q, Wu G, Guo WQ, Wang WW, et al. Influence factors of ultrasonic elastography for measurement of shear wave velocity. Chinese Journal of Medical Imaging 2014; 22: 697700.

[22] Karlas T, Pfrepper C, Wiegand J, Wittekind C, Neuschulz M, Mössner J, et al. Acoustic radiation force impulse imaging (ARFI) for non-invasive detection of liver fibrosis: examination standards and evaluation of interlobe differences in healthy subjects and chronic liver disease. Scand J Gastroenterol 2011; 46: 1458-1467.

[23] Toshima T, Shirabe K, Takeishi K, Motomura T, Mano Y, Uchiyama $\mathrm{H}$, et al. New method for assessing liver fibrosis based on acoustic radiation force impulse: a special reference to the difference between right and left liver. $J$ Gastroenterol 2011; 46: 705-711.

[24] Lupsor M, Badea R, Stefanescu H, Sparchez Z, Branda H, Serban A, et al. Performance of a new elastographic method (ARFI technology) compared to unidimensional transient elastography in the noninvasive assessment of chronic hepatitis C. Preliminary results. $J$ Gastrointestin Liver Dis 2009; 18: 303-310.

[25] Dillman JR, Heider A, Bilhartz JL, Smith EA, Keshavarzi N, Rubin JM, et al. Ultrasound shear wave speed measurements correlate with liver fibrosis in children. Pediatr Radiol 2015; 45: 1480-1488.

[26] Ma X, Zhan W, Zhang B, Wei B, Wu X, Zhou M, et al. Elastography for the differentiation of benign and malignant liver lesions: a metaanalysis. Tumour Biol 2014; 35: 4489-4497.

[27] Sporea I, Sirli R, Bota S, Popescu A, Sendroiu M, Jurchis A. Comparative study concerning the value of acoustic radiation force impulse elastography (ARFI) in comparison with transient elastography (TE) for the assessment of liver fibrosis in patients with chronic hepatitis B and C. Ultrasound Med Biol 2012; 38: 13101316.

[28] Nightingale K, Palmeri M, Trahey G. Analysis of contrast in images generated with transient acoustic radiation force. Ultrasound Med Biol 2006; 32: 61-72.

[29] Qiao XH, Xing JF. Principle and clinical application of shear wave ultrasound elastography. Chinese Interventional Imaging and Therapeutics 2015, 12: 512-515.

[30] Barr RG, Ferraioli G, Palmeri ML, Goodman ZD, Garcia-Tsao G, Rubin J, et al. Elastography assessment of liver fibrosis: society of radiologists in ultrasound consensus conference statement. Radiology 2015; 276: 845-861.

[31] Samir AE, Dhyani M, Vij A, Bhan AK, Halpern EF, Méndez-Navarro $\mathrm{J}$, et al. Shear-wave elastography for the estimation of liver fibrosis in chronic liver disease: determining accuracy and ideal site for measurement. Radiology 2015; 274: 888-896.

[32] Franchi-Abella S, Corno L, Gonzales E, Antoni G, Fabre M, Duco $\mathrm{B}$, et al. Feasibility and diagnostic accuracy of supersonic shear-wave elastography for the assessment of liver stiffness and liver fibrosis in children: a pilot study of 96 patients. Radiology 2016; 278: 554-562.

[33] Selmi B, Engelmann G, Teufel U, El Sakka S, Dadrich M, Schenk JP. Normal values of liver elasticity measured by real-time tissue elastography (RTE) in healthy infants and children. $J$ Med Ultrason 2014; 41: 31-38.

[34] Deffieux T, Gennisson JL, Bousquet L, Corouge M, Cosconea S, Amroun D, et al. Investigating liver stiffness and viscosity for fibrosis, steatosis and activity staging using shear wave elastography. J Hepatol 2015; 62: 317-324.

[35] Dietrich CF, Bamber J, Berzigotti A, Bota S, Cantisani V, Castera L, et al. EFSUMB Guidelines and Recommendations on the Clinical Use of Liver Ultrasound Elastography, Update 2017 (Long Version). Ultraschall Med 2017; 38: e16-e47.

[36] Naganuma H, Ishida H, Uno A, Nagai H, Kuroda H, Ogawa M. Diagnostic problems in two-dimensional shear wave elastography of the liver. World J Radiol 2020; 12: 76-86.

[37] Xie LT, Yan CH, Zhao QY, He MN, Jiang TA. Quantitative and noninvasive assessment of chronic liver diseases using twodimensional shear wave elastography. World J Gastroenterol 2018; 24: $957-970$.

[38] Fraquelli M, Rigamonti C, Casazza G, Donato MF, Ronchi G, Conte $\mathrm{D}$, et al. Etiology-related determinants of liver stiffness values in chronic viral hepatitis B or C. J Hepatol 2011; 54: 621-628. 